\title{
Vacancy defect control of colossal thermopower in $\mathrm{FeSb}_{2}$
}

\author{
Qianheng Du $\mathbb{D}^{1,2 凶}$, Lijun Wu $\mathbb{D}^{1}$, Huibo Cao $\mathbb{D}^{3}$, Chang-Jong Kang $\mathbb{D}^{4}$, Christie Nelson ${ }^{5}$, Gheorghe Lucian Pascut ${ }^{4,6}$, Tiglet Besara $\mathbb{D}^{7,9}$, \\ Theo Siegrist ${ }^{7,8}$, Kristjan Haule $\mathbb{I D}^{4}$, Gabriel Kotliar ${ }^{1,4}$, Igor Zaliznyak $\mathbb{D}^{1}$, Yimei Zhu ${ }^{1}$ and Cedomir Petrovic (D) ${ }^{1,2 凶}$
}

Iron diantimonide is a material with the highest known thermoelectric power. By combining scanning transmission electron microscopic study with electronic transport neutron, X-ray scattering, and first principle calculation, we identify atomic defects that control colossal thermopower magnitude and nanoprecipitate clusters with Sb vacancy ordering, which induce additional phonon scattering and substantially reduce thermal conductivity. Defects are found to cause rather weak but important monoclinic distortion of the unit cell Pnnm $\rightarrow$ Pm. The absence of Sb along [010] for high defect concentration forms conducting path due to Fe $d$ orbital overlap. The connection between atomic defect anisotropy and colossal thermopower in FeSb $\mathrm{Saves}_{2}$ the way for the understanding and tailoring of giant thermopower in related materials.

npj Quantum Materials (2021)6:13; https://doi.org/10.1038/s41535-020-00308-z

\section{INTRODUCTION}

Thermoelectric materials exploit thermoelectric effect where temperature difference is converted into electric power and vice versa $^{1,2}$. High operating temperatures are favorable for significant figure of merit $\mathrm{ZT}=S^{2} \sigma T / \kappa$, where $T$ is temperature, $S$ is thermopower, and $\sigma(\kappa)$ are electrical (thermal) conductivities. When operating temperatures are not high, material must maximize its thermoelectric power factor $\left(S^{2} \sigma\right)$ where $S$ provides considerable contribution, whereas in cryogenic environment electronic correlations could also be significant ${ }^{3-8}$.

The iron diantimonide is a correlated narrow-gap semiconductor $^{9,10}$. It also features colossal thermopower $S$ and highest known thermoelectric power factor at cryogenic temperatures; whereas both electronic diffusion and phonon-drag mechanism have been proposed, reported maximum $S$ values vary between 0.1 and $50 \mathrm{mV} \mathrm{K}^{-1}$ and in some crystals quasi-one-dimensional (quasi-1D) conductivity has been observed ${ }^{9,11-18}$. Interstitial Fe atoms in the unit cell that create impurity states connected with phonon drag have been postulated but never observed ${ }^{16}$. The connection with fine details of crystal structure is not understood since, for example, $\mathrm{FeAs}_{2}$ features an order of magnitude higher thermal conductivity and should have higher thermopower within the phonon drag mechanism when compared to $\mathrm{FeSb}_{2}$, but experiments show $S$ maxima about six times smaller in iron diarsenide ${ }^{14}$.

Atomic defects have been used to enhance high-temperature thermoelectric performance by lowering phonon thermal conductivity in the figure of merit ${ }^{19,20}$. Defect optimization in semiconductors is important in a wide range of technology-enabling materials ${ }^{21}$. In this work, for the first time, we present the direct visualization of structural defects in iron diantimonide and we show that colossal thermopower arises from Sb vacancies in the crystal structure. Moreover, thermopower, quasi-1D conductivity, and thermal conductivity are tunable by $\mathrm{Sb}$ defects and phase separation clusters with Sb vacancies of several nanometer size. Our results uncover hitherto unknown relation between crystal structure and colossal thermopower, thus providing basis for the comprehensive understanding of the nanostructural aspects related to the colossal thermopower control in iron diantimonide.

\section{RESULTS AND DISCUSSION}

\section{Sample characterization}

We first fabricated several crystals from $\mathrm{Sb}$ melt using different decanting methods, purity of raw elements, and crucibles (see Supplementary Note 4 and Table 7). Then we picked two crystals: one with expected high purity (S8) and the other with expected low purity (S3). To shed light on the atomic-scale structure, we performed high-resolution scanning transmission electron microscopy (STEM) with a high-angle annular dark field (HAADF) detector as its contrast is proportional to $Z^{1.7}$ along the atom column, where $Z$ is the atomic number. Figure $1 a, b$ show the STEM-HAADF images taken from S3 and S8 crystals, respectively. The strong and weak dots in the images correspond to $\mathrm{Sb}$ and Fe atoms, respectively. The atomic arrangement in the images is consistent with the $\mathrm{FeSb}_{2}$ structure with Pnnm symmetry, as shown in the insets where the atomic projections are embedded in the magnified image. Due to the $Z$-contrast nature of the STEM-HAADF image, the peak intensity of each dot can be used to count the atoms along the column ${ }^{22}$. Higher peak intensity indicates more atoms along the column, while weaker peak intensity indicates less atoms, thus more vacancies along the column. It is seen that the peak intensity of Fe is quite uniform in crystal S8 (Fig. 1b), indicating the relative uniform distribution of Fe. However, the peak intensity of Fe varies in crystal S3, e.g., there are less Fe in area I than that in area II, as shown in the magnified image in the insets of Fig. 1a. We also observe $\mathrm{Sb}$ intensity variation, indicating the variation of $\mathrm{Sb}$ occupancies. The Sb occupancies or vacancies can be better resolved by refining each $\mathrm{Sb}$ column peak with the second-order polynomial function. Figure 1c, d shows the peak intensity maps of Sb for crystals S3 and S8, respectively. There are four Sb atoms in a $\mathrm{FeSb}_{2}$ unit cell. In crystal S3, the Sb peak intensity is the same in some area (inset III in Fig. 1c), consistent with the Pnnm symmetry.

\footnotetext{
${ }^{1}$ Condensed Matter Physics and Materials Science Department, Brookhaven National Laboratory, Upton, NY, USA. ${ }^{2}$ Department of Materials Science and Chemical Engineering, Stony Brook University, Stony Brook, NY, USA. ${ }^{3}$ Neutron Scattering Division, Oak Ridge National Laboratory, Oak Ridge, TN, USA. ${ }^{4}$ Department of Physics and Astronomy, Rutgers University, Piscataway, NJ, USA. ${ }^{5}$ National Synchrotron Light Source II, Brookhaven National Laboratory, Upton, NY, USA. ${ }^{6}$ MANSiD Research Center and Faculty of Forestry, Stefan Cel Mare University (USV), Suceava, Romania. ${ }^{7}$ National High Magnetic Field Laboratory, Florida State University, Tallahassee, FL, USA. ${ }^{8}$ Department of Chemical and Biochemical Engineering, FAMU-FSU College of Engineering, Tallahassee, FL, USA. ${ }^{9}$ Present address: Department of Physics, Astronomy \& Materials Science, Missouri State University, Springfield, MO 65897, USA. ${ }^{凶}$ email: qdu@bnl.gov; petrovic@bnl.gov
} 

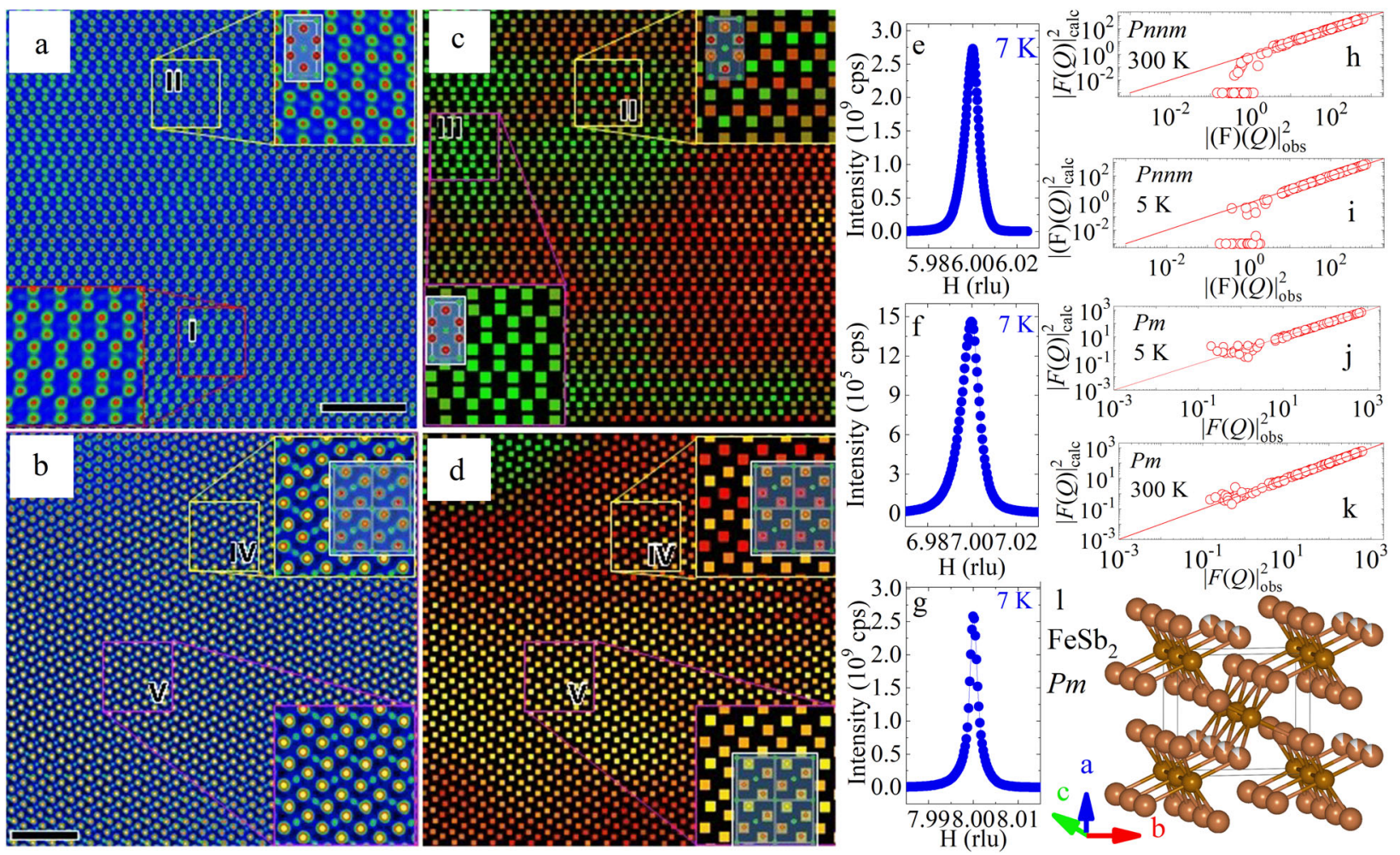

Fig. 1 Atomic defects in FeSb, and crystal structure distortion. a, b STEM-HAADF image of crystal S3 (a) viewed along Pnnm [100] direction and crystal S8 viewed along Pnnm [001] direction (b). The insets are the magnified images from areas I and II in a (areas IV and V in $\mathbf{b}$ ) with the Pnnm [100] in a (Pnnm [001] projection in b) projection of the structure embedded. Scale bar $2 \mathrm{~nm}$. The contrast is approximately proportional to $Z^{1.7}$ along the atomic column, thus the dots with strong and weak contrast correspond to $\mathrm{Sb}$ and Fe column, respectively. Red and green spheres represent $\mathrm{Sb}$ and Fe, respectively. $\mathbf{c}, \mathbf{d}$ Sb peak intensity maps refined from $\mathbf{a}$ and $\mathbf{b}$, respectively. Each square represents an Sb column with intensity increasing in black-blue-green-orange-yellow-white order. The insets are magnified maps from areas II to V. Note that the smooth Sb peak intensity oscillation from top to bottom in $\mathbf{b}$ and $\mathbf{d}$ could be attributed to thickness variation. $\mathbf{e}-\mathbf{g}$ Synchrotron $\mathrm{X}$-ray diffraction scans of crystal S8. $H$ is the Miller index of the diffraction from (H00). Pnnm-forbidden peak is observed at [100] wavevector (f), about 3 orders of magnitude weaker than nearby Bragg peaks. Pnnm (h, i) and Pm (j, $\mathbf{k})$ unit cell refinements obtained in single-crystal neutron diffraction experiment on low thermopower crystal, confirming structural distortion. I Pm unit cell of FeSb ${ }_{2}$ induced by atomic defects. Central octahedral atom Fe (dark) is surrounded by Sb (light). The defects are preferably on Sb11 atomic sites (white arrow-marked light balls).

In the other area, however, the $\mathrm{Sb}$ peak intensity changes periodically and orders along Pnnm [010] direction, as shown in the inset II in Fig. 1C, where two Sb peaks (orange squares) are stronger than the other two Sb (green squares) within the unit cell. This indicates the ordering of $\mathrm{Sb}$ vacancies and reduction of the Pnnm symmetry in this area. In [100] projection, orange and red spheres represent high and low Sb occupancy, respectively, embedded in the inset II. The size of the phase separation clusters with $\mathrm{Sb}$ vacancy ordering is about a few nanometers. These clusters are similar to the nanoprecipitates observed in $\mathrm{PbTe}-\mathrm{AgSbTe}_{2}$ system ${ }^{23,24}$. They induce additional phonon scattering, thus reduce the thermal conductivity and phonon mean free path (MFP) of crystal S3. For crystal S8, the Sb vacancy ordering is observed in nearly all locations and also along [010] direction. In area IV (Fig. 1d), two $\mathrm{Sb}$ peaks (orange) are stronger than the other two Sb (red). While in the area V, one Sb peak (yellow square) is stronger than the other three $\mathrm{Sb}$ (orange squares).

Sb vacancy ordering results in a Pnnm-forbidden peak observed in single-crystal synchrotron X-ray diffraction (Fig. 1e-g) of crystal S8, consistent with weak structural distortion observed in neutron diffraction measurements (Fig. 1h-k). Neutron diffraction shows the presence of weak $(h, 0, l), h+I=$ odd and $(0, k, l), k+I=$ odd type Bragg reflections, which are forbidden in Pnnm crystal structure previously refined for the stoichiometric $\mathrm{FeSb}_{2}{ }^{9,10,25}$. The presence of these forbidden reflections hints that the symmetry of the crystal lattice is lower. Comprehensive structural refinement (Fig. $1 \mathrm{~h}-\mathrm{k}$ ) and also Supplementary Note 1 and Tables 1-4 indicate that one of the two Sb sites, which are equivalent in Pnnm space group, shows displacive monoclinic distortions and site deficiency. This induces change in the structural symmetry $(P n n m \rightarrow P m)$, making two Sb sites inequivalent, i.e., Sb1 site is fully occupied while Sb11 site contains substantial number of vacancies that do not change with temperature (Fig. 1I). We find that the Sb11 site is 0.82 (2) occupied while both Fe sites are 0.94(2) occupied; the chemical vacancies do not change with temperature, whereas intensity of Pnnm-forbidden peaks at $300 \mathrm{~K}$ is $2 / 3$ of that at $5 \mathrm{~K}$ (see Supplementary Note 1 and Tables 1-4).

\section{Electrical and thermal transport properties}

Having established the presence of atomic vacancies, next we focus on electrical and thermal transport properties of crystals S3 and S8 as well as of six additional crystals engineered to have different defect content. Figure $2 a-c$ presents electronic and thermal transport difference among all eight iron diantimonide crystals. Crystals S7 and S8 have about 1-2 order of magnitude higher electrical resistivity when compared to crystals S1, S2, and S3 in the temperature region 10-20 K (Fig. 2a). Moreover, crystals S1, S2, and S3 have clear weak (semi)metallic resistivity in 60-300 K temperature region. Low-temperature thermopower $S$ shows large variation (Fig. $2 b$ ); $|S|$ maxima change by several orders of magnitude from S1 $\left(14 \mu \mathrm{V} \mathrm{K}^{-1}\right)$ to $\mathrm{S} 8\left(20 \mathrm{mV} \mathrm{K}^{-1}\right)$. Figure $2 c$ shows thermal conductivity $K$ vs temperature for all crystals. The small $k(T)$ maxima are coincident with small thermopower, consistent with phonon-drag mechanism ${ }^{15-17}$. 

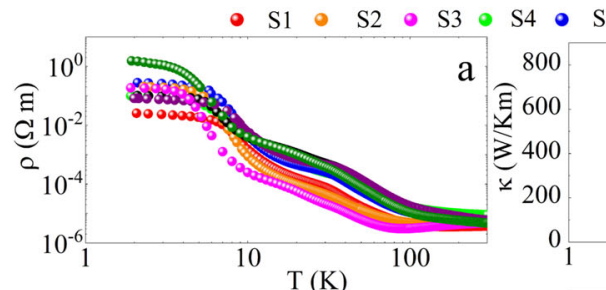

$\mathrm{S} 5 \cdot \mathrm{S} 6 \quad \bullet \quad \mathrm{S} 7 \quad \bullet \quad \mathrm{S} 8$
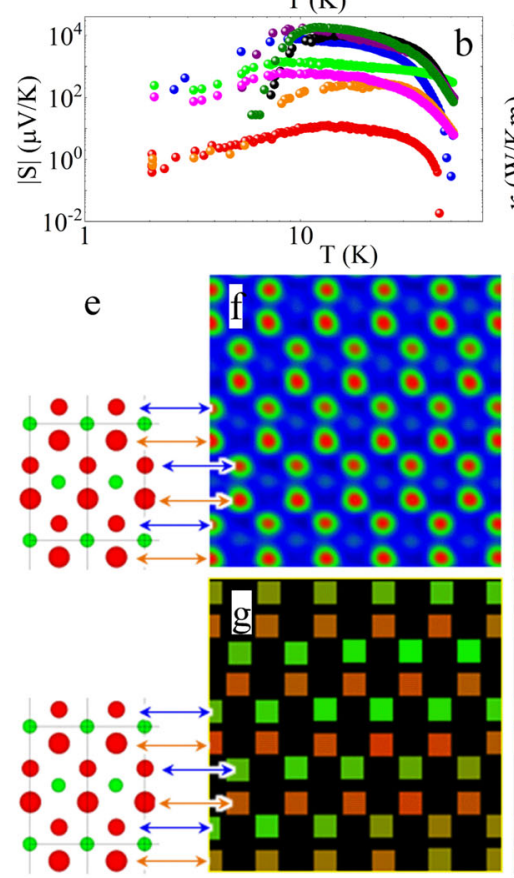


0.96



ㄴ.1 0.90

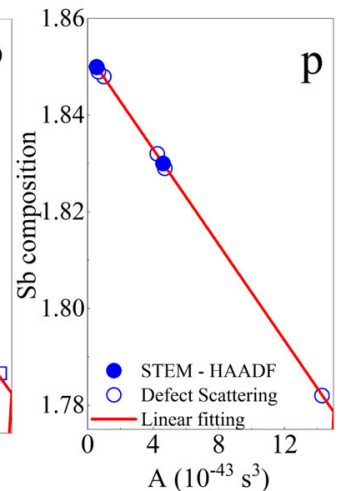

$\mathrm{p}$
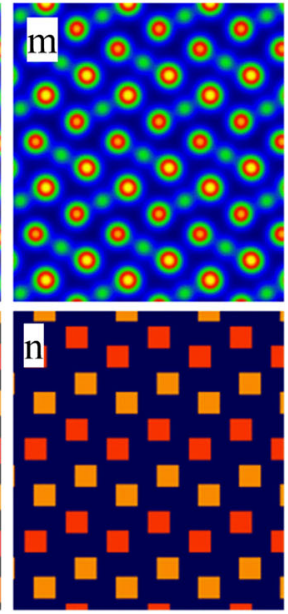

Fig. 2 Relationship between the defect content and transport properties. Temperature dependence of the a electrical resistivity, b thermopower, and $\mathbf{c}$ thermal conductivity for the investigated iron diantimonide crystals with different defect content (see text). $\mathbf{d}$ Thermal conductivity $<50 \mathrm{~K}$. The solid lines show the fitting by Callaway model. e [100] projection of crystal S3 with green and red spheres representing $\mathrm{Fe}$ and $\mathrm{Sb}$ atoms. The occupancy of $\mathrm{Fe}$ is 0.93 . The occupancy of $\mathrm{Sb}$ at the row indicated by blue arrow lines is 0.85 (small spheres), while that indicated by brown arrow lines are 0.98 (large spheres). f Magnified image from area II in Fig. 1 a. $\mathbf{g}$ Sb peak intensity map refined from $\mathbf{f}$. $\mathbf{h}$ Simulated image calculated based on multislice method with frozen phonon approximation using structure model in e. i Sb peak intensity map from $\mathbf{h}$. From the simulation, the contrast at Sb site changes with the occupancy of Sb, e.g., the contrast of Sb at the row indicated by blue arrow lines is weaker than that at the row indicated by brown arrow lines. $\mathbf{j}$ [001] projection of crystal S8. The occupancy of $\mathrm{Fe}$ is 0.95 . The occupancy of $\mathrm{Sb}$ at the row indicated by blue arrow lines is 0.88 , while that indicated by brown arrow lines is 0.97 . $\mathbf{k}$ Magnified image from area IV in Fig. 1 b. I Sb peak intensity map refined from $\mathbf{k}$. $\mathbf{m}$ Simulated image based on structure model in $\mathbf{j}$. $\mathbf{n}$ Sb peak intensity map from $\mathbf{m}$. From the simulation, the contrast at $\mathrm{Sb}$ site changes with the occupancy of $\mathrm{Sb}$, e.g., the contrast of $\mathrm{Sb}$ at the row indicated by blue arrow lines is weaker than that at the row indicated by brown arrow lines. o, p present composition of all the investigated crystals in a-c. $A$ is the fitting parameter in Callaway model as discussed in the main text.

In what follows, we focus on the deviation from the ideal 1:2 stoichiometry. The contribution of different relaxation processes to $\tau$ and their difference among investigated crystals in the simplest form can be assessed from:

$$
\begin{aligned}
\tau^{-1} & =\tau_{\mathrm{B}}^{-1}+\tau_{1}^{-1}+\tau_{\mathrm{U}}^{-1} \\
& =\frac{v}{L}+A \omega^{4}+B \omega^{2} T \mathrm{e}^{-\frac{\theta_{\mathrm{D}}}{T}}
\end{aligned}
$$

where $\tau_{\mathrm{B}}, \tau_{\mathrm{D}}$, and $\tau_{U}$ are the relaxation times for boundary scattering, impurities or defect scattering, and Umklapp processes, respectively ${ }^{26-28}$. The $L, A$, and $B$ are fitting parameters in the fit of the experimental lattice thermal conductivity using Callaway model (Fig. 2d):

$$
K_{\mathrm{L}}=\frac{k_{\mathrm{B}}}{2 \pi^{2} v_{\mathrm{s}}}\left(\frac{k_{\mathrm{B}}}{\hbar}\right)^{3} T^{3} \int_{0}^{\frac{\theta_{\mathrm{D}}}{T}} \frac{\tau x^{4} \mathrm{e}^{x}}{\left(\mathrm{e}^{x}-1\right)^{2}} d x
$$

In this model, $x=\frac{\hbar \omega}{k_{B} T}$ is dimensionless, $\omega$ is the phonon frequency, $k_{\mathrm{B}}$ is the Boltzmann constant, $\hbar$ is the Plank constant, $\theta_{\mathrm{D}}$ is the Debye temperature, and $v_{\mathrm{s}}$ is the velocity of sound. Fit parameters are listed in Table 1. Since Umklapp scattering processes are of importance at high temperature, we mainly focus on parameters $A$ and $L$. In crystals S5, S6, S7, and S8 with large phonon MFP, surface scattering is more important when compared to S1, S2, S3, and S4 where the impurity scattering processes are much stronger as inferred from $A$ (Table 1), which features inverse scaling with $\tau$. The decrease of MFP in the investigated crystals is clearly related to the defect scattering of phonons.

We note that, in the Callaway model, the parameter $\tau_{B}=L / V$ represents the relaxation time determined by boundary scattering. The $L$ is the phonon MFP at low temperature in the boundary scattering regime ${ }^{29}$, which is different from grain size. For the rodlike sample with the square cross-section and infinite length, it depends on the side dimension only. For the finite length and rectangular cross-section, there are correction factors, i.e., it not only depends on two side dimensions but also on their ratio and sample length ${ }^{29,30}$.

From the data collected on crystal S3 (Fig. 1h-k) and Supplementary Table 3, the refined crystal stoichiometry is $\mathrm{Fe}_{0.93} \mathrm{Sb}_{1.83}$. STEM-HAADF image simulations (Fig. 2e-n) show $\mathrm{Fe}_{0.93} \mathrm{Sb}_{1.83}$ for crystal S3 (in good agreement with neutron refinement) and $\mathrm{Fe}_{0.95} \mathrm{Sb}_{1.85}$ for crystal $\mathrm{S}^{22}$. 
Q. Du et al.

Table 1. Fitting parameters of the Callaway model for the thermal conductivity and the stoichiometry for all the samples (see text).

\begin{tabular}{lllllllll}
\hline Sample no. & $A$ & $L$ & $B$ & $S b$ composition & Fe compsition & Stoichiometry & Normalized stoichiometry & EDX \\
\hline S1 & $14.30(0.45)$ & $5(1)$ & $10.5(1.0)$ & $1.782(1)$ & $0.883(1)$ & $\mathrm{Fe}_{0.883} \mathrm{Sb}_{1.782}$ & $\mathrm{FeSb}_{2.018}$ & $\mathrm{FeSb}_{1.969}$ \\
S2 & $4.71(0.09)$ & $10(1)$ & $3.3(0.3)$ & $1.829(1)$ & $0.929(1)$ & $\mathrm{Fe}_{0.929} \mathrm{Sb}_{1.829}$ & $\mathrm{FeSb}_{2.07}$ \\
S3 & $4.60(0.20)$ & $5(1)$ & $6.0(0.5)$ & 1.830 & 0.930 & $\mathrm{Fe}_{0.93} \mathrm{Sb}_{1.830}$ & $\mathrm{FeSb}_{1.968}$ & \\
S4 & $4.25(0.08)$ & $10(1)$ & $3.9(0.3)$ & $1.832(1)$ & $0.932(1)$ & $\mathrm{Fe}_{0.932} \mathrm{Sb}_{1.832}$ & $\mathrm{FeSb}_{1.966}$ & $\mathrm{FeSb}_{2.10}$ \\
S5 & $0.99(0.07)$ & $40(3)$ & $7.0(0.7)$ & $1.848(1)$ & $0.948(1)$ & $\mathrm{Fe}_{0.948} \mathrm{Sb}_{1.848}$ & $\mathrm{FeSb}_{1.949}$ & \\
S6 & $0.66(0.04)$ & $37(3)$ & $7.5(0.6)$ & $1.849(1)$ & $0.949(1)$ & $\mathrm{Fe}_{0.949} \mathrm{Sb}_{1.849}$ & $\mathrm{FeSb}_{1.948}$ & $\mathrm{FeSb}_{1.95}$ \\
S7 & $0.57(0.03)$ & $85(5)$ & $2.9(0.3)$ & $1.850(1)$ & $0.950(1)$ & $\mathrm{Fe}_{0.950} \mathrm{Sb}_{1.850}$ & $\mathrm{FeSb}_{1.947}$ & $\mathrm{FeSb}_{1.947}$ \\
S8 & $0.55(0.05)$ & $100(5)$ & $3.1(0.3)$ & 1.850 & 0.950 & $\mathrm{Fe}_{0.950} \mathrm{Sb}_{1.850}$ & $\mathrm{FeSb}_{2.03}$ \\
\hline
\end{tabular}

Note: error bars for EDX are: S1: $\pm 0.07, \mathrm{~S} 3: \pm 0.03, \mathrm{~S} 5: \pm 0.05$, and S7: \pm 0.02 . And the units in Callaway model fit for $A, L$, and $B$ are $10^{-43} \mathrm{~s}^{3}, 10^{-5} \mathrm{~m}$, and $10^{-18} \mathrm{~S}$ $\mathrm{K}^{-1}$, respectively.

Next, from the thermal conductivity fits (Table 1), we note differences among crystals due to defect scattering, given by parameter $A$ in the Callaway model. From the changes in parameter $A$, we estimate the defects content, i.e., the stoichiometry of all investigated crystals by assuming linear relation between defect scattering and defect concentration. The results are shown in Fig. 2o, p and in Table 1. The relative stoichiometry ratio of $\mathrm{Sb}$ :Fe differs from the ideal stoichiometric 2:1 in all crystals in linear manner, albeit with differences that are $<2.3$ atomic \%.

Thermally activated resistivity $>50 \mathrm{~K}$ (Fig. 2a) stems from the intrinsic energy gap; however, the resistivity shoulder in the region of $S_{\max }$ around $10 \mathrm{~K}$ is a fingerprint of the in-gap impurity states that couple to phonon drag ${ }^{16,18,31,32}$. Magnetoresistance (MR) is strong in the temperature range where such states are dominant in electronic transport; a single in-gap band gives only one peak in $M^{16}$. High-thermopower crystals S6 and S8 show one MR peak but in low-thermopower crystals S1 and S3 a broad MR is observed (Fig. 3a), suggesting a rather broad distribution of multiple in-gap states ${ }^{16}$. A low-temperature Curie tail present in crystal S3 (Fig. 3b) implies higher low-temperature magnetic moment associated with low Sb purity ${ }^{33}$, consistent with importance of $\mathrm{Sb}$ defects. Figure $3 \mathrm{c}$ shows heat capacity of all crystals. We observe a trend of the slope change associated with Debye temperature. In general, vacancy formation energy is related to Debye temperature: $T_{D}=C \frac{E_{V}^{1 / 2}}{M^{1 / 2} \Omega^{1 / 3}}$, where $C$ is a constant, $E_{\mathrm{v}}$ is the vacancy formation energy, and $M$ and $\Omega$ are atomic mass and volume, respectively ${ }^{34,35}$. Low vacancy formation energy is consistent with higher number of vacancies for low thermopower crystals (e.g., S3) when compared to highthermopower crystals (e.g., S8). Results are summarized in Supplementary Note 2 and Table 5.

The Pnnm FeSb6 octahedra are edge-sharing along the shortest lattice parameter $3.194 \AA$. Closer inspection of $P m$ unit cell shows that the Sb11 atomic sites (Fig. 4a) are also separated by the lattice parameter length $3.194 \AA$ along the edge-sharing octahedral direction. However, weak metallic conductivity at high temperature for low-thermopower crystals (Fig. 2a) is along the orthogonal, $6.536 \AA$ lattice parameter direction where distance of Sb11 to Fe is shorter (b-direction in Fig. $4 a)^{9,36}$. This is consistent with TEM-observed vacancy order direction (Fig. 1a-d) and quasi1D conductivity in optics ${ }^{37,38}$. Occupancy of Fe $d$ orbital $\left(d^{n}\right)$ translates into different unit cell parameter along the $d$ orbital overlap. Higher (lower) occupancy corresponds to larger (shorter) unit cell parameter ${ }^{39,40}$. Detailed comparison of crystals S3 and S8 shows longer $b$ lattice parameter in S3 (see Supplementary Note 3 and Table 6), implying differences in $\mathrm{Fe} d$ orbital occupation. A picture emerges where Sb11 vacancy defects create Fe-derived conducting in-gap states due to short Fe-Sb11 hopping distance (Fig. 4a). Low vacancy formation energy, i.e., higher vacancy content in low-thermopower crystals such as S3


Fig. 3 The signature of in-gap impurity states. a Magnetoresistance of low-thermopower (S1, S3) and high-thermopower crystals (S6, S8). b Comparison of magnetic susceptibility of crystals S3 and S8. c Low-temperature heat capacity for all the investigated crystals.

(Fig. 3c), promotes stronger quasi-1D Fe $d$ orbital overlap due to the absence of Sb along [010], weak metallicity, and metal-insulator transition on cooling ${ }^{12}$.

\section{Band structure calculations}

GW+DMFT calculations (see Supplementary Note 5) show that bands associated with quasi-1D dispersion along $6.536 \AA$ are $\mathrm{Fe}$ derived: bottom of the conduction band is dominated by Fe $x y$ orbital, whereas top of the valence bands is dominated by Fe $x z / y z$ bands $^{38}$. First-principle calculation results (Fig. $4 b-h$ ) confirm that structure distortion to the $P m$ space group is energetically favorable in Sb-deficient Pnnm FeSb 2 unit cell. However, the Sb vacancies also induce Fe dangling bonds that might influence electronic structure through conducting impurity band at the Fermi level for high $\mathrm{Sb}$ defect concentration.

To explore how $\mathrm{Sb}$ atom vacancy and its ratio affect the electronic structure of $\mathrm{FeSb}_{2}$, we studied two cases of Sb vacancy ratios: one and two $\mathrm{Sb}$ vacancies in a $2 \times 2 \times 3$ super-cell of $\mathrm{FeSb}_{2}$. To simulate the experimental situation, we only consider $\mathrm{Sb}$ vacancies at the Sb11 site. Hence, Sb vacancies for both cases 

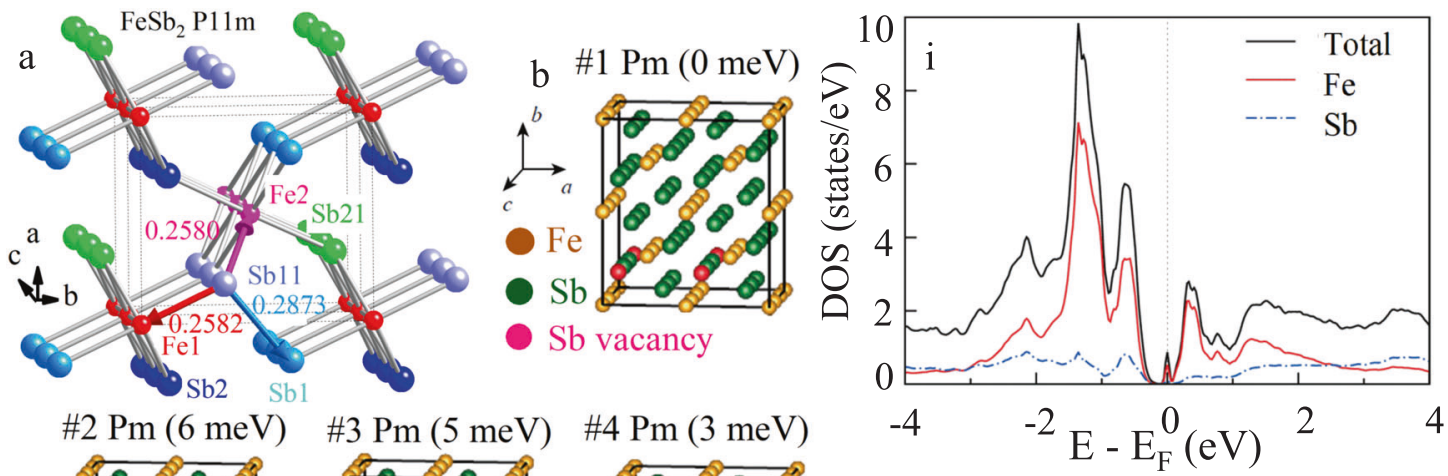

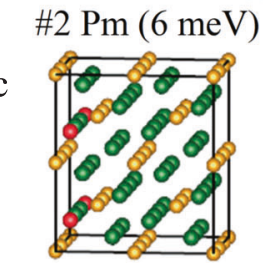

\#5 Pm (1 meV)

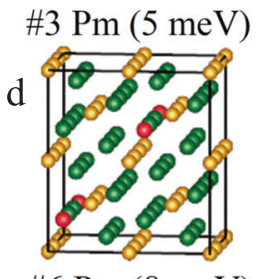

$\mathrm{f}$

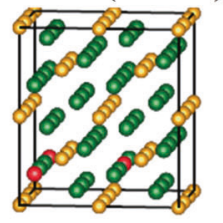

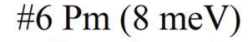

$\mathrm{g}$

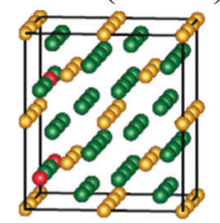

\#4 Pm (3 meV)

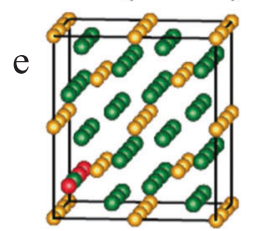

\#7 Pm $(6 \mathrm{meV})$

$\mathrm{h}$
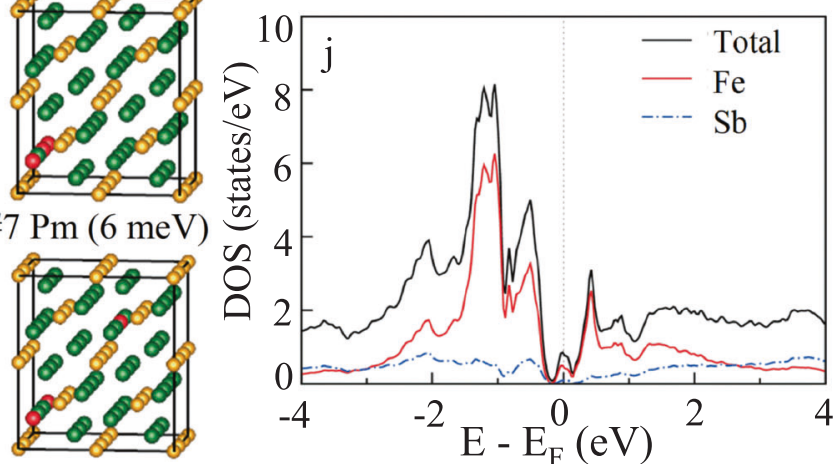

Fig. $4 \mathrm{Pm}$ space group and corresponding band structure calculation. a Crystal structure of $\mathrm{FeSb}_{2}$ in the $\mathrm{Pm}$ space group. Note that $\mathrm{Sb}$ deficient Sb11 atomic site are apical for Fe1, whereas they are within octahedral plane for Fe2. Bond distances to Fe1, Fe2, and Sb1 are shown in $\mathrm{nm}$. Sb11 atomic sites are separated by the unit cell distance along Pmc axis (3.194 $\AA$ ), whereas they are closer to Sb1 sites along the $b$-axis (2.878 ̊̊). b-h Different arrangements of two Sb vacancies in the supercell and their energetics. The arrangement consistent with the Pm space group has the lowest energy, in agreement with the experiment. Projected density of states for $\mathbf{i}$ one and $\mathbf{j}$ two Sb vacancies. Notice that the antimony vacancy gives rise to an iron impurity band.

occupy the Sb11 site only, and they correspond to 91.7 and $83.3 \%$ of Sb11 occupancy, respectively, where the case of two Sb vacancies is close to the experimental Sb11 occupancy, $82 \%$ at $T$ $=300 \mathrm{~K}$. For the case of one Sb vacancy, there is only one symmetrically non-equivalent configuration for introducing one $\mathrm{Sb}$ vacancy at the $\mathrm{Sb} 11$ site in the $2 \times 2 \times 3$ super-cell. On the other hand, for the case of two Sb vacancies, there are seven symmetrically non-equivalent configurations. We have considered all of the seven configurations and found that the configuration with two Sb vacancies is the most energetically stable (Fig. 4b). From now on, we only focus on the most stable configuration for each $\mathrm{Sb}$ vacancy ratio.

Figure $4 \mathrm{i}, \mathrm{j}$ shows the density of states of the most stable configurations for both $\mathrm{Sb}$ vacancy ratios. The $\mathrm{Sb}$ vacancies give rise to Fe dangling bonds that lead to metallic impurity bands at the Fermi level. The impurity bands have a dominant $d$ orbital character of Fe possessing the dangling bonds. We would like to note that the density-functional theory calculation with the modified Becke-Johnson exchange potential method gives a clear bulk gap of $\sim 0.25 \mathrm{eV}$ in $\mathrm{FeSb}_{2}$ without any vacancies. As depicted in Fig. 4j, the case of two Sb vacancies shows broader bandwidth of the metallic impurity band than that of one $\mathrm{Sb}$ vacancy, indicating that, as the Sb vacancy ratio increases, the metallic impurity band is more dispersive. The case of one $\mathrm{Sb}$ vacancy does not show the quasi-1D conductivity. Therefore, we conclude that larger Sb atom vacancy content induces more anisotropy in the electronic structure and triggers the quasi-1D conducting path.

Now, we turn our attention to the thermoelectric power of $\mathrm{FeSb}_{2}$. In the experiment, the maximum value of thermoelectric power decreases as the energy of vacancy formation is lowered, i.e., with higher number of vacancies (Figs. $2 b$ and $3 c$ ). This is consistent with a two-band model study with an ionized impurity donor state ${ }^{41}$. Hence, the increased $\mathrm{Sb}$ atom vacancies lower thermoelectric power, in agreement with the STEM-HAADF study. It would be of interest to investigate point defects and atomic structure of other marcasite materials with high-thermopower values, such as $\mathrm{CrSb}_{2}{ }^{42,43}$.

In summary, we have achieved point defect control of thermoelectricity and have directly observed atomic structure of colossal thermopower material iron diantimonide. Sb defects induce monoclinic distortion and generate the in-gap states with Fe $3 d$ orbital character, which, for sufficiently high number of defects, result in nanoprecipitates with Sb vacancy ordering and quasi-1D conducting path. Our study demonstrates the potential of low-level atomic defects to control thermopower magnitude and quasi-1D electronic conduction. This paves the way toward computational predictions of colossal thermopower induced by atomic point defect engineering.

\section{METHODS}

\section{Crystal synthesis}

Single crystals of $\mathrm{FeSb}_{2}$ were grown as described before ${ }^{9,10,12}$, albeit with different starting purity of $\mathrm{Fe}$ and $\mathrm{Sb}$ and with variable methods of crystal decanting from $\mathrm{Sb}$ liquid. Whereas within one single batch thermopower values $\left|S_{\max }\right|$ exhibit variations, especially for low-purity starting materials such as $\mathrm{Fe} 99.5 \%$ and $\mathrm{Sb} 99.99 \%$ and lower, the use of high-purity starting materials, such as Fe $99.997 \%$ and Sb $99.9999 \%$, elimination of quartz wool in excess Sb decanting and use of filter screw-top crucibles developed for flux crystal growth above the quartz melting point ${ }^{44}$ reduces the variation. This produces $\mathrm{FeSb}_{2}$ crystals with about $5-20 \mathrm{mV} \mathrm{K}^{-1}$ thermopower peak within single batch. On the other hand, we note that, among different batches with variable starting materials purity and decanting method, $\rho(T)$ and $S(T)$ measured on same crystal exhibit close correspondence to values presented in Fig. 2 in the main text. 


\section{Transport, thermal, and magnetic measurements}

Crystals were oriented using a Laue camera and cut along the $b$-axis for magnetization, resistivity, and thermopower measurement in a Quantum Design MPMS-5 and PPMS-9.

\section{Scanning tunneling microscopy}

STEM-HAADF imaging and electron diffraction were performed using the double aberration-corrected JEOL-ARAM200CF microscope with a coldfield emission gun and operated at $200 \mathrm{keV}$. The images are filtered in frequency space by applying periodic mask to remove noise.

\section{$X$-ray and neutron diffraction}

Synchrotron single-crystal X-ray diffraction was performed at NSLS-II beamline 4-ID, using a photon energy of $11.44 \mathrm{keV}$ with an incident beam intensity of $3 \times 10^{12}$ photons $\mathrm{s}^{-1}$. Single-crystal neutron diffraction was performed at the HB3A four-circle diffractometer (FCD) equipped with a two-dimensional Anger camera detector at the High Flux Isotope Reactor at the Oak Ridge National Laboratoray. Neutron wavelength of $1.005 \AA$ was used from the bent perfect Si-331 monochromator ${ }^{45}$. Room temperature laboratory single-crystal X-ray diffraction was performed at NHMFL Tallahassee using an Oxford Diffraction Xcalibur 2 charge-coupled device FCD with graphite-monochromated Mo Ka radiation.

\section{DATA AVAILABILITY}

The data that support the findings of this study are available from the corresponding authors upon reasonable request.

Received: 10 August 2020; Accepted: 22 December 2020; Published online: 12 February 2021

\section{REFERENCES}

1. Bell, L. E. Cooling, heating, generating power, and recovering waste heat with thermoelectric systems. Science 321, 1457-1461 (2008).

2. Snyder, G. J. \& Toberer, E. S. Complex thermoelectric materials. Nat. Mater. 7, 105-114 (2008).

3. Heremans, J. P. Introduction to cryogenic solid state cooling. Proc. SPIE 9821 , $98210 \mathrm{G}$ (2016).

4. He, J., Kanatzidis, M. G. \& Dravid, V. P. High performance bulk thermoelectric via a panoscopic approach. Mater. Today 16, 166-176 (2013).

5. Hébert, S. et al. Searching for new thermoelectric materials: some examples among oxides, sulfides and selenides. J. Phys. Condens. Matter 28, 013001 (2016).

6. Tomczak, J. M., Haule, K. \& Kotliar, G. Signatures of electronic correlations in iron silicide. Proc. Natl Acad. Sci. USA 109, 3243-3246 (2012).

7. Palsson, G. \& Kotliar, G. Thermoelectric response near the density driven Mott transition. Phys. Rev. Lett. 80, 4775-4778 (1998).

8. Koshibae, W. \& Maekawa, S. Effects of spin and orbital degeneracy on the thermopower of strongly correlated systems. Phys. Rev. Lett. 87, 236603 (2001).

9. Petrovic, C. et al. Anisotropy and large magnetoresistance in the narrow-gap semiconductor FeSb 2 . Phys. Rev. B 67, 155205 (2003).

10. Petrovic, C. et al. Kondo insulator description of spin state transition in $\mathrm{FeSb}_{2}$. Phys. Rev. B 72, 045103 (2005).

11. Bentien, A. et al. Colossal Seebeck coefficient in strongly correlated semiconductor FeSb 2 . Eur. Phys. Lett. 80, 17008 (2007).

12. Jie, Q. et al. Electronic thermoelectric power factor and metal-insulator transition in FeSb 2 . Phys. Rev. B 86, 115121 (2012).

13. Sun, P. et al. Huge thermoelectric power factor: FeSb $b_{2}$ versus $\mathrm{FeAs}_{2}$ and RuSb $\mathrm{B}_{2}$. Appl. Phys. Express 2, 091102 (2009).

14. Sun, P. et al. Narrow band gap and enhanced thermoelectricity in FeSb 2 . Dalton Trans. 39, 1012-1019 (2010).

15. Tomczak, J. M. et al. Thermopower of correlated semiconductors: application to $\mathrm{FeAs}_{2}$ and FeSb $\mathrm{F}_{2}$. Phys. Rev. B 82, 085104 (2010).

16. Battiato, M., Tomczak, J. M., Zhong, Z. \& Held, K. Unified picture for the colossal thermopower in FeSb 2. Phys. Rev. Lett. 114, 236603 (2015).

17. Takahashi, H. et al. Colossal Seebeck effect enhanced by quasi-ballistic phonons dragging massive electrons in $\mathrm{FeSb}_{2}$. Nat. Commun. 7, 12732 (2016).

18. Matsuura, H., Maebashi, H., Ogata, M. \& Fukuyama, H. Effect of phonon drag on Seebeck coefficient based on linear response theory: application to $\mathrm{FeSb}_{2}$. J. Phys. Soc. Jpn. 88, 074601 (2019).
19. Biswas, K. et al. Strained endotaxial nanostructures with high thermoelectric figure of merit. Nat. Chem. 3, 160-166 (2011).

20. Kim, T. K. et al. Strain-mediated point defects in thermoelectric p-type bismuth telluride polycrystalline. Nano Energy 55, 486-493 (2019).

21. Walsh, A. \& Zunger, A. Instilling defect tolerance in new compounds. Nat. Mater. 16, 964-967 (2017)

22. LeBeau, J. M., Findlay, S. D., Allen, L. J. \& Stemmer, S. Standardless atom counting in scanning transmission electron microscopy. Nano Lett. 10, 4405-4408 (2010).

23. Hsu, K. F. et al. Cubic $\mathrm{AgPb}_{m} \mathrm{SbTe}_{2+m}$ : bulk thermoelectric materials with high figure of merit. Science 303, 818-821 (2004).

24. $\mathrm{Ke}, \mathrm{X}$. et al. Microstructure and a nucleation mechanism for nanoprecipitates in PbTe-AgSbTe 2 . Phys. Rev. Lett. 103, 145502 (2009).

25. Hägg, G. X-ray Studies on the Binary Systems of Iron with Nitrogen, Phosphorus, Arsenic, Antimony and Bismuth (Almqvist \& Wiksell, Uppsala, 1929).

26. Wang, K., Hu, R., Warren, J. \& Petrovic, C. Enhancement of the thermoelectric properties in doped $\mathrm{FeSb}_{2}$ bulk crystals. J. Appl. Phys. 112, 013703 (2012).

27. Callaway, J. Model for lattice thermal conductivity at low temperatures. Phys. Rev. 113, 1046-1051 (1959).

28. Onn, D. G. et al. Some aspects of the thermal conductivity of isotopically enriched diamond single crystals. Phys. Rev. Lett. 68, 2806-2809 (1992).

29. Inyushkin, A. V. et al. Thermal conductivity of high purity synthetic single crystal diamonds. Phys. Rev. B 97, 144305 (2018).

30. McCurdy, A. K., Maris, H. J. \& Elbaum, C. Anisotropic heat conduction in cubic crystals in the boundary scattering regime. Phys. Rev. B 2, 4077-4083 (1970).

31. Sun, P. et al. Highly dispersive electron relaxation and colossal thermoelectricity in the correlated semiconductor FeSb 2 . Phys. Rev. B 88, 245203 (2013).

32. Takahashi, H., Okazaki, R., Yasui, Y. \& Terasaki, I. Low-temperature magnetotransport of the narrow-gap semiconductor FeSb 2 . Phys. Rev. B 84, 205215 (2011).

33. Takahashi, H., Yasui, Y., Terasaki, I. \& Sato, M. Effects of ppm-level imperfection on the transport properties of $\mathrm{FeSb}_{2}$ single crystals. J. Phys. Soc. Jpn. 80, 054708 (2011).

34. Mukherjee, K. Monovacancy formation energy and Debye temperature of closepacked metals. Philos. Mag. 12, 915-918 (1965).

35. March, N. H. Vacancy formation energy and Debye temperature in close packed metals. Phys. Lett. 20, 231-232 (1966).

36. Hu, R. et al. Colossal positive magnetoresistance in a doped nearly magnetic semiconductor. Phys. Rev. B 77, 085212 (2008).

37. Perucchi, A. et al. Optical investigation of the metal-insulator transition in $\mathrm{FeSb}_{2}$. Eur. Phys. J. B 54, 175-183 (2008).

38. Homes, C. C. et al. Unusual electronic and vibrational properties in the colossal thermopower material FeSb 2 . Sci. Rep. 8, 11692 (2018).

39. Hull, G. W. \& Hulliger, F. CuSe 2 , a marcasite type superconductor. Nature 220, 257-258 (1968)

40. Hulliger, F. in Structure and Bonding, Vol. 4 83-229 (Springer, Berlin, 1968).

41. Kang, C. J. \& Kotliar, G. Study for material analogs of FeSb $b_{2}$ : material design for thermoelectric materials. Phys. Rev. Mater. 2, 034604 (2018).

42. Sales, B. C. et al. Transport, thermal, and magnetic properties of the narrow-gap semiconductor $\mathrm{CrSb}_{2}$. Phys. Rev. B 86, 235136 (2012).

43. Du, Q., Guzman, D., Choi, S. \& Petrovic, C. Crystal size effects on giant thermopower in $\mathrm{CrSb}_{2}$. Phys. Rev. B 101, 035125 (2020).

44. Petrovic, C., Canfield, P. C. \& Mellen, J. Y. Growing intermetallic single crystals using in situ decanting. Philos. Mag. 92, 2448-2457 (2012).

45. Chakoumakos, B. et al. Four-circle single-crystal neutron diffractometer at the high flux isotope reactor. J. Appl. Crystallogr. 44, 655-658 (2011).

\section{ACKNOWLEDGEMENTS}

Work at Brookhaven National Laboratory is supported by the Office of Basic Energy Sciences, Materials Sciences and Engineering Division, U.S. Department of Energy under Contract No. DE-SC0012704 (Q.D., L.W., I.Z., Y.Z., and C.P.) and as a part of the Computational Materials Science Program (C.-J.K., G.L.P., K.H., and G.K.). This research used resources at the High Flux Isotope Reactor, a DOE Office of Science User Facility operated by the Oak Ridge National Laboratory. This research used beamline 4-ID of the National Synchrotron Light Source II, a U.S. Department of Energy (DOE) Office of Science User Facility operated for the DOE Office of Science by Brookhaven National Laboratory under Contract No. DE-SC0012704. A portion of this work was performed at the National High Magnetic Field Laboratory, which is supported by National Science Foundation Cooperative Agreement No. DMR-1644779 and the State of Florida.

\section{AUTHOR CONTRIBUTIONS}

C.P. designed research. Q.D. and C.P. made crystals. Q.D. carried out transport, magnetization, and thermal measurements and analysis with C.P. TEM measurement and analysis was done by L.W. and Y.Z. C.N. performed synchrotron X-ray diffraction measurement. H.C. and I.Z. carried out neutron diffraction measurements and solved 
new structure with input from L.W. and Y.Z. T.B. and T.S. carried out laboratory singlecrystal measurements. C.-J.K., G.L.P., K.H., and G.K. carried out first-principle calculations. C.P. supervised the project and wrote the paper with Q.D. and with input from L.W., I.Z., C.-J.K., and G.K. The manuscript reflects contribution and ideas of all authors.

\section{COMPETING INTERESTS}

The authors declare no competing interests.

\section{ADDITIONAL INFORMATION}

Supplementary information The online version contains supplementary material available at https://doi.org/10.1038/s41535-020-00308-z.

Correspondence and requests for materials should be addressed to Q.D. or C.P.

Reprints and permission information is available at http://www.nature.com/ reprints
Publisher's note Springer Nature remains neutral with regard to jurisdictional claims in published maps and institutional affiliations. (i) Open Access This article is licensed under a Creative Commons Attribution 4.0 International License, which permits use, sharing, adaptation, distribution and reproduction in any medium or format, as long as you give appropriate credit to the original author(s) and the source, provide a link to the Creative Commons license, and indicate if changes were made. The images or other third party material in this article are included in the article's Creative Commons license, unless indicated otherwise in a credit line to the material. If material is not included in the article's Creative Commons license and your intended use is not permitted by statutory regulation or exceeds the permitted use, you will need to obtain permission directly from the copyright holder. To view a copy of this license, visit http://creativecommons. org/licenses/by/4.0/.

(c) The Author(s) 2021 